\title{
Correction to: MAP kinase signaling and inhibition in melanoma
}

\author{
R. J. Sullivan · K. Flaherty
}

Published online: 3 March 2021

(c) The Author(s), under exclusive licence to Springer Nature Limited 2021

Correction to: Oncogene

https://doi.org/10.1038/onc.2012.345

Following the publication of this article, the authors noted a missing reference. The reference is listed below. The paragraphs where the reference is cited should appear as follows (bold indicates changes to the originally published article):

First paragraph on 2377: Whether ABT-263 in combination with MEK or selective BRAF inhibitors will improve clinical outcomes is remains unknown, though appears worth exploring in an early-phase clinical trial, as we have argued previously [97].

Third paragraph on 2377: In addition to combination therapy with molecularly targeted agents that inhibit signaling induced by selective BRAF inhibition or promote apoptosis, we continue to believe that another promising approach to maximizing the benefit of BRAF or MEK inhibitors is to combine these agents with immunotherapy [97].

Third paragraph on 2377: We maintain that these findings provide a rationale for a study evaluating the safety and efficacy of selective BRAF inhibition in combination with immunotherapy, including ipilimumab, MDX1106 and possibly high-dose IL2 [97].

Fourth paragraph on 2377: It had been hoped for many years that the growing understanding of the molecular pathways involved in melanoma development and the increasing availability of specific inhibitors of these pathways would enable the rational development of future therapies, as we have noted previously [97].

Fifth paragraph on 2377: Importantly, the great majority of these patients will acquire resistance to BRAF inhibitors, and as the mechanisms of this resistance become elucidated, we still hold the view that the development of combination trials of novel molecularly targeted therapies can be expected [97].

\section{Reference}

97. Sullivan RJ, Flaherty KT. BRAF in melanoma: pathogenesis, diagnosis, inhibition, and resistance. J Skin Cancer. 2011;2011:423239. 